\title{
The Effect of Vibration and Low-Frequency Audio on Full-Body Haptic Sensations
}

\author{
Ernst Kruijff*, Christina Trepkowski \\ Bonn-Rhein-Sieg University of Applied Sciences \\ Sankt Augustin, Germany
}

\author{
Robert W. Lindeman \\ Worcester Polytechnic Institute \\ Worcester, MA, USA
}

\section{Introduction}

We report on two experiments that deploy low-frequency audio and strong vibrations to induce haptic-like sensations throughout the human body. Vibration is quite frequently deployed in immersive systems, for example to provide collision feedback, but its actual effects are not well understood [Kruijff \& Pander 2005; Kruijff et al. 2015]. The starting point of our experiments was a study by Rasmussen [Rasmussen 1982], which found that different vibration frequencies were experienced differently throughout the body. We will show how vibrations affect sensations throughout the body and may provide some directional cues to some parts of the body, yet also illustrate the difficulties.

\section{Experiments and results}

Inspired by vibration literature, we wanted to verify how the use of different frequencies affects haptic-like sensations. Vibration stimuli were presented through a bass-shaker with a frequency range of 1-20,000 Hz. In Experiment 1, one bass-shaker was either mounted underneath a chair or under a foot support, while in Experiment 2, two bass-shakers were mounted underneath two foot supports that were fixed on a wooden floor plate to avoid them moving around, and to keep a constant distance between them. Furthermore, in Experiment 1 we deployed a custom-built large subwoofer, with a frequency range of $5 \mathrm{~Hz}$ and up. For both experiments, we produced eight-second WAV files (square sinusoidal waves at $5 \mathrm{~Hz}$ steps) consisting of the stimuli to be provided to the user. During each experiment, we asked the participant to rate the extent of haptic-like feedback (low, medium, strong) in their legs, lower body, chest, arms, and head.

For Experiment 1, data from 31 participants was analyzed, Vibration frequency (supplied through the bass-shakers) showed a significant main effect on both feet $(F(5.24,78.57)=70.56, p<$ $.001)$ and lower body point of entry conditions $(F(3.99,55.80)=$ $85.06, p<.001)$. Point of entry (feet versus lower body) had a significant main effect on the vibration intensity $(F(1,29)=4.76$, $p<.05)$. Ratings show vibration strength is related to the distance from the point of entry; the body parts closest are rated highest. Vibration frequency showed a significant main effect on both feet $(F(5.24,78.57)=70.56, p<.001)$ and lower body point of entry

*e-mail: ernst.kruijff@h-brs.de

Permission to make digital or hard copies of all or part of this work for personal or classroom use is granted without fee provided that copies are not made or distributed for profit or commercial advantage and that copies bear this notice and the full citation on the first page. Copyrights for components of this work owned by others than ACM must be honored. For all other uses, contact the Owner/Author.

Copyright is held by the owner/author(s)

VRST '15, November $13-15,2015$, Beijing, China.

ACM 978-1-4503-3990-2/15/11.

http://dx.doi.org/10.1145/2821592.2821626 conditions $(F(3.99,55.80)=85.06, p<.001)$, with clear peaks around 20 and $60 \mathrm{~Hz}$. For Experiment 2, data from 16 participants was analyzed, and revealed that sensation strength was affected by side (point of entry), body part and vibration frequency ( $F$ $\left.(12.44,186.61)=3.59, p<.001, \eta^{2}=.19\right)$. When stimulating one side, mostly the leg and the lower body on that side are affected by vibration. Simple effect comparisons confirmed the visual impression for the point of entry. For right foot stimulation, we found peak frequencies were at 5,60 and $140 \mathrm{~Hz}$. At these frequencies, the sensation in the right leg was rated significantly higher than the other body parts $(p<.001)$. At 60 and $140 \mathrm{~Hz}$, also the right lower body was rated significantly higher than the other body parts (except the feet). Left leg stimulation showed slightly different results: peak frequencies were at 20,60 and 130 $\mathrm{Hz}$. At all frequencies, the left leg was rated higher than the other body parts $(p<.001)$, while also the left lower body was rated higher than most body parts, but not higher than head and left arm. Finally, for both feet, we found peaks at 8, 50 and $120 \mathrm{~Hz}$.

Results showed there are clear vibration frequency peaks for haptic sensations supplied by the bass-shakers, yet, that it is difficult to target isolated sensations in specific parts of the body other than those closest to the point of entry, as legs and lower body were mostly rated significantly higher than the other body parts. Hence, we could not replicate results found by Rasmussen. In line with previous work on whole-body vibration, point of entry indeed had a strong effect on the sensation. However, most vibration was transmitted to the lower part of the body during lower body stimulation, instead of upwards; theoretically, the waist is also quite close to the lower body, yet never received significantly higher ratings. Comparing the various peaks from both experiments illustrates that based on the point of entry, different frequencies will result in different sensations. As such, when designing systems, it is recommended to fine-tune the frequencies to come to the optimal result. Finally, we could not find a significant effect of audio on the haptic-like sensations, though users rated audio positively on the overall effect.

In conclusion, we think that a hybrid approach will work best. For example, wearable vibration, combined with floor vibration, and shakers embedded in game controllers might be a successful approach. This would provide support for different uses of vibration, such as contact cues, proximity cues, and informational cues, similar to different visual elements used as functional cues.

\section{References}

KRUiJfF, E. AND PANDER, A. 2005. Experiences of using Shockwaves for Haptic Sensations. Proceedings of $3 D$ user interface workshop, IEEE VR 2005. .

Kruijff, E., Marquardt, A., Trepkowski, C., Schild, J., AND HinKENJANN, A. 2015. Enhancing User Engagement in Immersive Games through Multisensory Cues. In Proceedings of VS-GAMES.

Rasmussen, G. 1982. Human Body Vibration Exposure and its Measurement. Brüel \& Kjaer Technical Review 1, 1-35. 